\title{
ORIGINAL ARTICLE STIM1, a direct target of microRNA-185, promotes tumor metastasis and is associated with poor prognosis in colorectal cancer
}

\author{
This article has been corrected since publication and a corrigendum has also been published
}

\author{
Z Zhang ${ }^{1}$, X Liu', B Feng ${ }^{1}$, N Liu', Q Wu, Y Han, Y Nie, K Wu, Y Shi and D Fan
}

\begin{abstract}
STIM1 (stromal interaction molecule 1), an endoplasmic reticulum $\mathrm{Ca}^{2+}$ sensor that triggers the store-operated $\mathrm{Ca}^{2+}$ entry activation, has recently been implicated in cancer progression. However, the role of STIM1 in the progression and metastasis of colorectal cancer (CRC) has not been addressed. In this study, we confirmed increased expression of STIM1 in highly invasive CRC cell lines. Enhanced expression of STIM1 promoted CRC cell metastasis in vitro and in vivo, whereas silencing of STIM1 with small interfering RNA resulted in reduced metastasis. Ectopic expression of STIM1 in CRC cells induced epithelial-to-mesenchymal transition (EMT), whereas silencing of STIM1 had the opposite effect. Furthermore, STIM1 expression was markedly higher in CRC tissues than in adjacent noncancerous tissues. STIM1 overexpression correlated with poor differentiation and higher tumor node metastasis stage. CRC patients with positive STIM1 expression had poorer prognoses than those with negative STIM1 expression. Moreover, STIM1 was found to be a direct target of miR-185, a microRNA (miRNA) that has not previously been reported to be involved in EMT, in both CRC tissues and cell lines. Taken together, these findings demonstrate for the first time that STIM1 promotes metastasis and is associated with cancer progression and poor prognosis in patients with CRC. In addition, we show that expression of STIM1 is regulated by a posttranscriptional regulatory mechanism mediated by a new EMT-related miRNA. This novel miR-185-STIM1 axis promotes CRC metastasis and may be a candidate biomarker for prognosis and a target for new therapies.
\end{abstract}

Oncogene (2015) 34, 4808-4820; doi:10.1038/onc.2014.404; published online 22 December 2014

\section{INTRODUCTION}

Colorectal cancer (CRC) is one of the most prevalent carcinomas throughout the world, with an estimated one million new cases and half a million mortalities each year. ${ }^{1,2}$ Metastasis, one of the six initial cancer hallmarks, ${ }^{3}$ is a major cause of CRC-associated mortality. ${ }^{4}$ More than one-third of patients with CRC will ultimately develop metastatic disease, ${ }^{5}$ however, little is known about the exact molecular mechanisms underlying CRC metastasis. ${ }^{6}$ Understanding the key factors in these processes is crucial to the development of novel anticancer therapies.

In most types of cell, the ubiquitous second messenger $\mathrm{Ca}^{2+}$ is a critical regulator that mediates various cellular processes. ${ }^{7}$ In nonexcitable cells, the store-operated calcium influx (SOCE) is the major mechanism of $\mathrm{Ca}^{2+}$ entry. 8,9 Recent studies have identified two important molecules responsible for SOCE activation: STIM1 (stromal interaction molecule 1) and Orai1. ${ }^{8}$ When $\mathrm{Ca}^{2+}$ in the endoplasmic reticulum is depleted, STIM1 serves as a $\mathrm{Ca}^{2+}$ sensor and aggregates into multiple punctae that translocate within close proximity of the plasma membranes. ${ }^{10}$ Orai1, an essential poreforming component of the SOCE channel, translocates to these STIM1-containing structures during store depletion and open stomediate $\mathrm{Ca}^{2+}$ entry. ${ }^{11-13}$ Recently, STIM1 has been reported to have an important role in various pathophysiological conditions, such as the immune response, ${ }^{11,14}$ cardiovascular disease, ${ }^{15,16}$ sexual dysfunction ${ }^{17}$ and pulmonary disease. ${ }^{18}$ STIM1 was also found to be involved in cancer and was initially thought to work as a tumor suppressor, ${ }^{19}$ although subsequent data indicate an opposite function. ${ }^{20}$ More recently, Orai1 and STIM1 were shown to be essential for the migration of breast tumor cells. ${ }^{21}$ Another study found that STIM1 has an important role in the growth, migration and angiogenesis of cervical cancer. ${ }^{22}$ These studies suggest that STIM1 might have a critical role in cancer.

Epithelial-to-mesenchymal transition (EMT) is a morphogenetic process in which cells lose their epithelial characteristics such as cell polarity and cell-cell contact, and gain mesenchymal properties such as increased motility. EMT also endows cells with invasive properties, induces stem cell properties and prevents apoptosis and senescence. Morphologic evidence of EMT has been found at the invasive front of human tumors. ${ }^{23}$ EMT is viewed as an essential early step and a critical process during tumor metastasis. $^{24-27}$ Notably, several recent studies suggest that EMT initiated by Oct4 or Snail 1 in breast cancer may be associated with STIM1-directed $\mathrm{Ca}^{2}$ influx. ${ }^{21,23,28-30}$ These studies strongly suggest that STIM1 may promote cancer metastasis by regulating EMT.

MicroRNAs (MiRNAs) are noncoding small RNAs that posttranscriptionally regulate protein expression. ${ }^{31}$ Several studies have

State Key Laboratory of Cancer Biology and Xijing Hospital of Digestive Diseases, The Fourth Military Medical University, Xi'an, Shaanxi, People's Republic of China. Correspondence: Professor Y Shi or Professor D Fan, State Key Laboratory of Cancer Biology and Xijing Hospital of Digestive Diseases, The Fourth Military Medical University, 127 Chang Le West Road, Xi'an, Shaanxi 710032, People's Republic of China.

E-mail: shiyquan@fmmu.edu.cn or daimingfan@fmmu.edu.cn

${ }^{1}$ These authors contributed equally to this work.

Received 15 November 2013; revised 21 October 2014; accepted 4 November 2014; published online 22 December 2014 

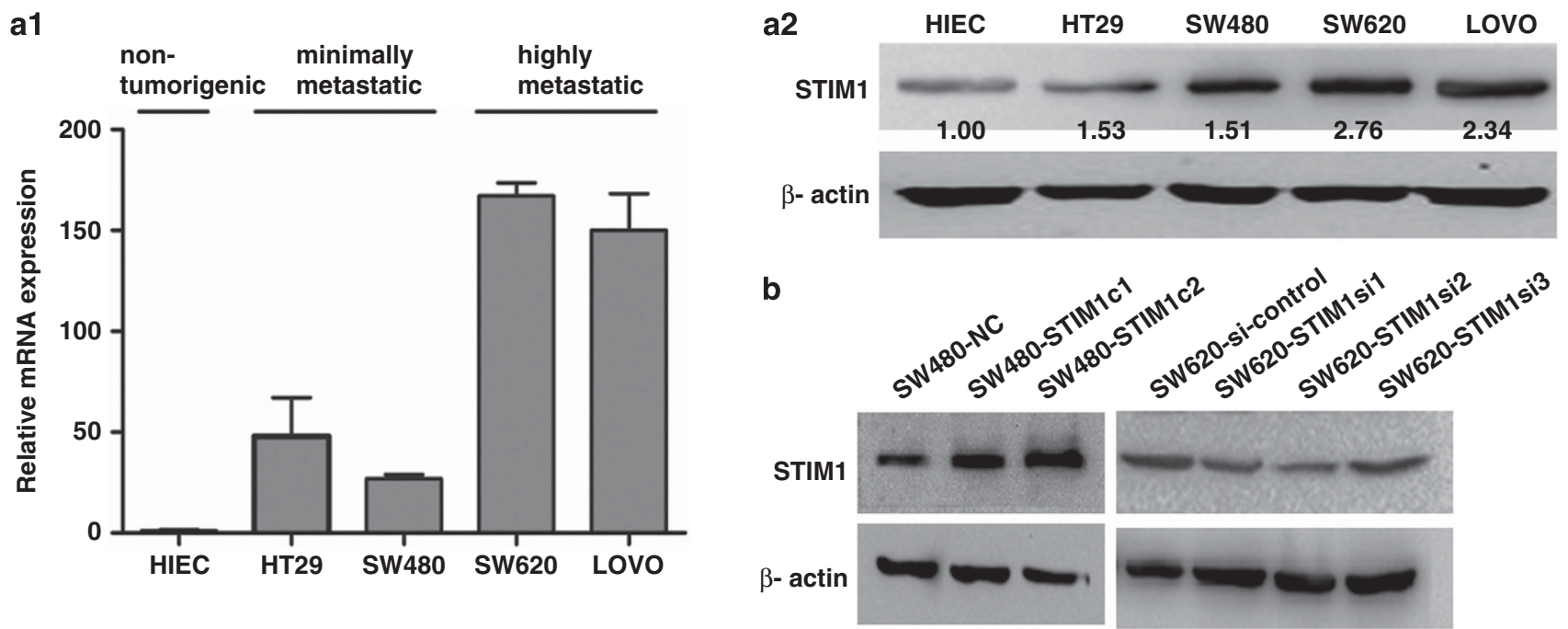

c1
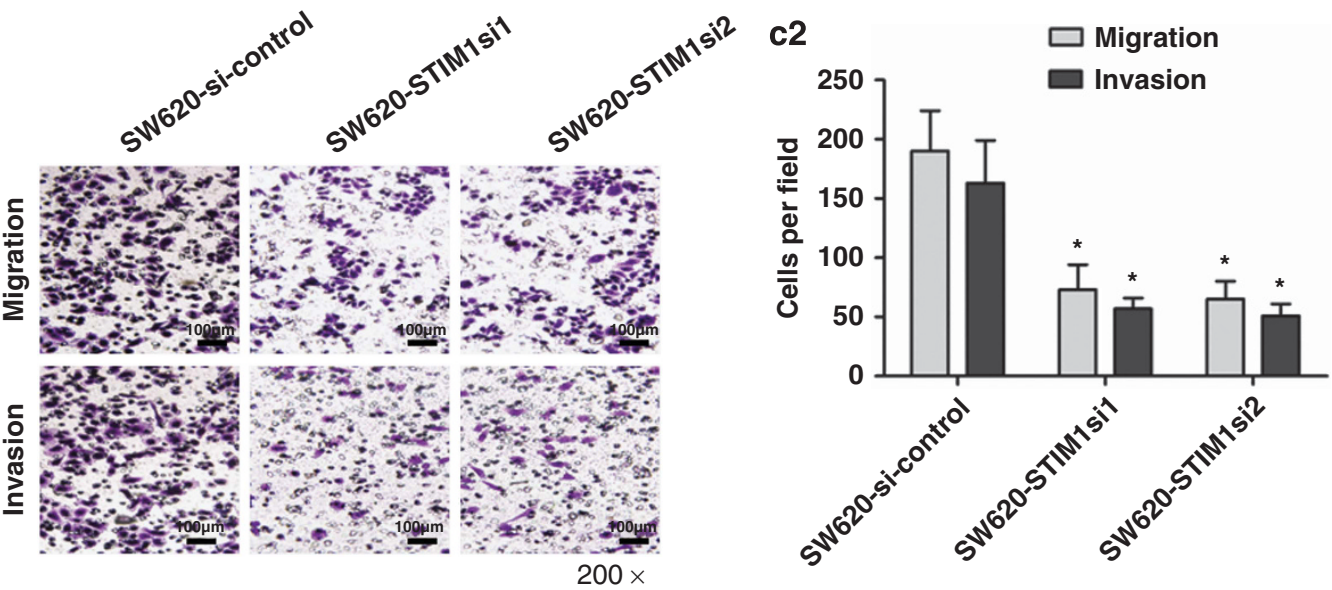

d1

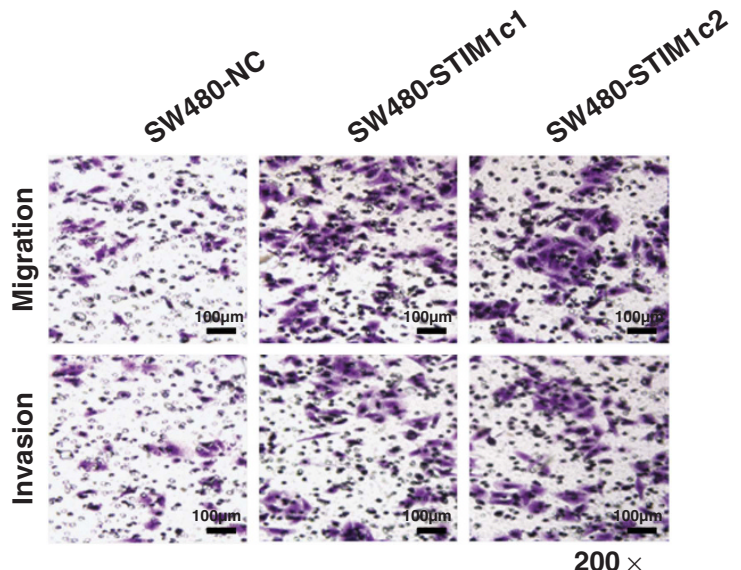

d2

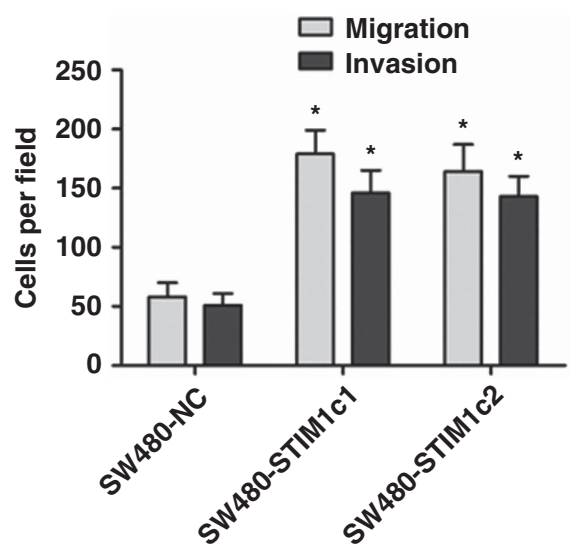

Figure 1. STIM1 promoted CRC cells invasion and metastasis in vitro. (a1) Expression of STIM1 in four human CRC cell lines relative to the normal human intestinal epithelial cell line (HIEC) was measured by qRT-PCR. (a2) Western blotting analysis of STIM1 expression in different CRC cell lines. (b) After cells were infected with LV-STIM1 (two clones, c1 and c2) or LV-STIM1 si-1, -2 or -3, the level of STIM1 protein expression was detected by western blotting analysis. (c) Invasion and migration assay of SW620 cells transfected with LV-STIM1si-1, LV-STIM1si-2 or a negative control. (c1) Representative fields of migration (left) or invasive (right) cells on membrane. (c2) Average cell number of invasion or migration per field from three independent experiments \pm s.e. ${ }^{*} P<0.05$. (d1 and d2) Invasion or migration assay of SW480 cells transfected with the LV-STIM1 expression vector (two clones, c1 and c2) or the control vector (NC). Scale bar, $100 \mu \mathrm{m}$. ${ }^{*} P<0.05$.

shown that miRNAs have an important role in cancer metastasis. ${ }^{32}$ Recently, some studies have highlighted the importance of miRNAs in the regulation of EMT. ${ }^{33,34}$ As both miRNAs and STIM1 may have important roles in EMT process, we are interested in whether STIM1 expression is regulated by certain miRNAs in cancer. Notably, a recent study has found that miR-195 directly regulates STIM1 mRNA decay in normal rat intestinal crypt cells. ${ }^{35}$ However, whether posttranscriptional regulation by certain 
miRNAs may represent one of the upstream regulation mechanisms of STIM1 expression in cancer metastasis remains unknown.

In this study, we provide the first evidence that STIM1 overexpression is associated with progression and poor prognosis in CRC. We show that STIM1 promotes CRC metastasis in vitro and in vivo. We further demonstrate that STIM1 is a direct functional target of miR-185, a new EMT-related miRNA identified in this study. Our results suggest that the miR-185/STIM1 signaling pathway might be a potential target for diagnosis and therapy in CRC.

\section{RESULTS}

STIM1 promotes migratory and invasive capacities of CRC cells in vitro and in vivo

To assess whether STIM1 expression correlates with the metastatic potential of CRC cells, we examined the expression of STIM1 in four human CRC cell lines (SW480, SW620, HT29 and LOVO) and in a normal human intestinal epithelial cell line. Basal expression levels of STIM1 were significantly increased in SW620 and LOVO cells, which have highly metastatic propensities, compared with either the poorly metastatic cell lines SW480 and HT29 or the

a

\begin{tabular}{lcc}
\multicolumn{3}{c}{ Incidence of metastasis in mice } \\
\hline & Lung metastasis & Liver metastasis \\
\hline SW480-NC & $2 / 10$ & $1 / 10$ \\
SW480-STIM1 c1 & $6 / 10$ & $5 / 10$ \\
SW480-STIM1 c2 & $7 / 10$ & $5 / 10$ \\
SW620-si-control & $8 / 10$ & $8 / 10$ \\
SW620-STIM1 si1 & $4 / 10$ & $3 / 10$ \\
SW620-STIM1 si2 & $3 / 10$ & $3 / 10$ \\
\hline
\end{tabular}
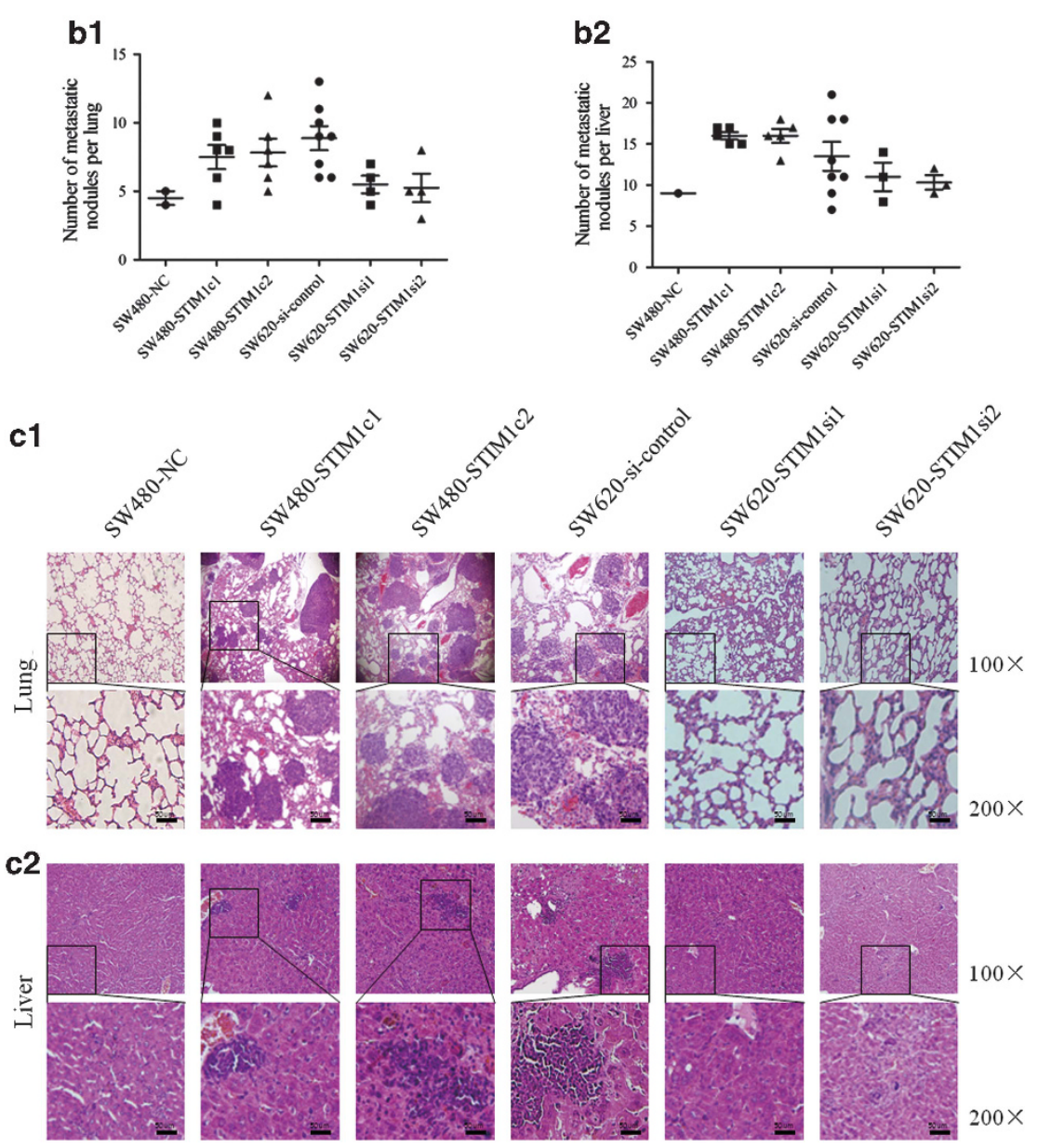

Figure 2. STIM1 promoted CRC cells invasion and metastasis in vivo. SW480 cells transfected with the LV-STIM1 expression vector (two clones, c1 and (2) or the control vector and SW620 cells transfected with LV-STIM1si-1, LV-STIM1si-2 or a negative control were injected into nude mice via the tail vein for an in vivo metastasis assay. Animals were killed 6 weeks after injection (a). Incidence of lung and liver metastasis in each group of nude mice. (b) Number of metastatic lung foci observed in each group. (b2) Number of metastatic liver foci observed in each group. (c1) Images showing representative hematoxylin and eosin staining of lung tissue samples from the different experimental groups. (c2) Images showing representative hematoxylin and eosin staining of liver tissue samples from the different experimental groups. Scale bar, $50 \mu \mathrm{m}$. 
immortalized cell line human intestinal epithelial cell line (Figures 1a1 and a2). The SW480 cell line was obtained from a primary CRC lesion, and the SW620 cell line was established from a lymph node $(\mathrm{LN})$ metastasis in the same patient a year later. ${ }^{36}$ Therefore, these cell lines have the same genetic background but different metastatic potential. ${ }^{37}$ To explore whether STIM1 affects

a

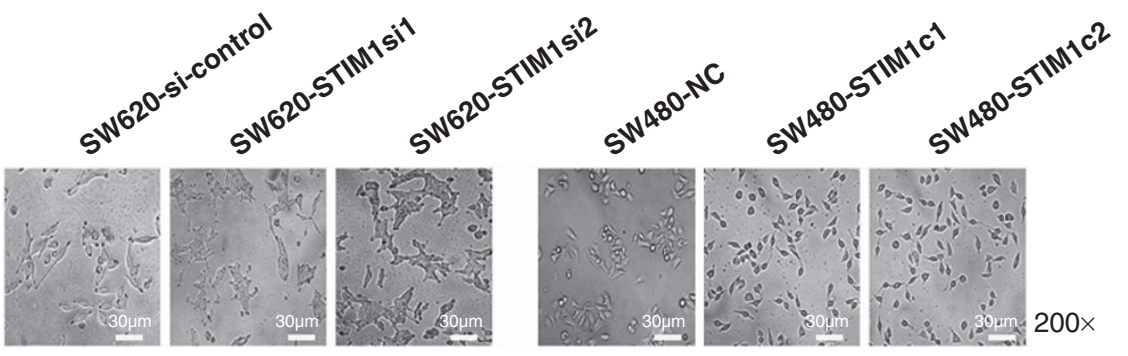

b

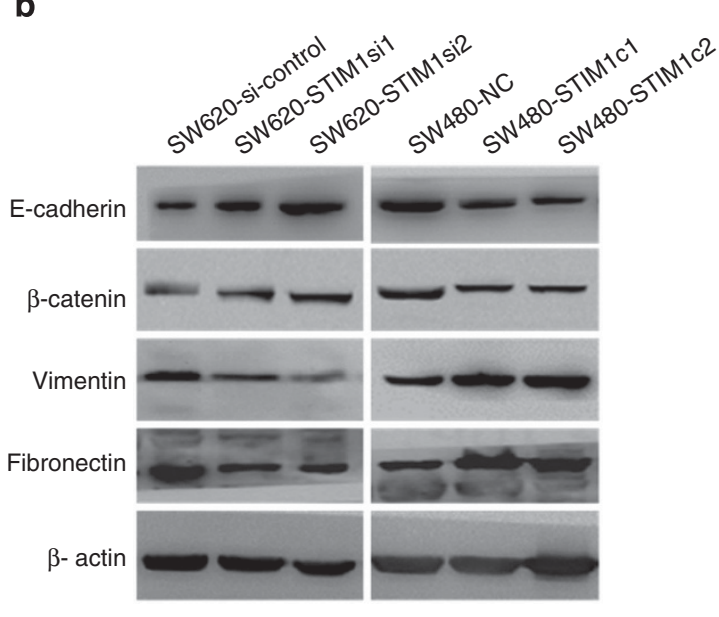

C
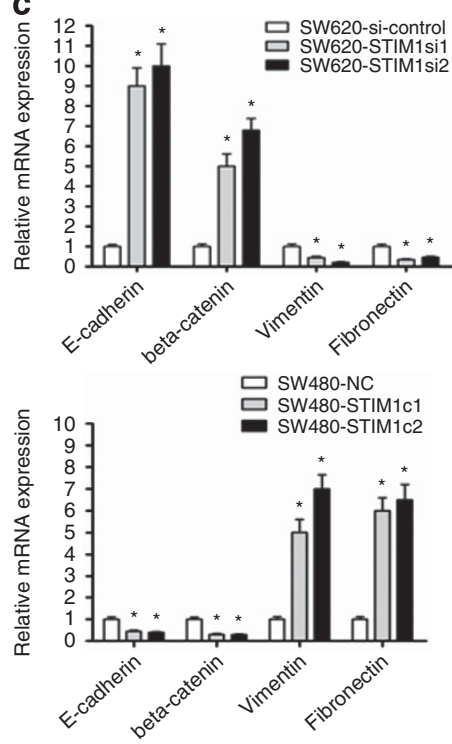

d

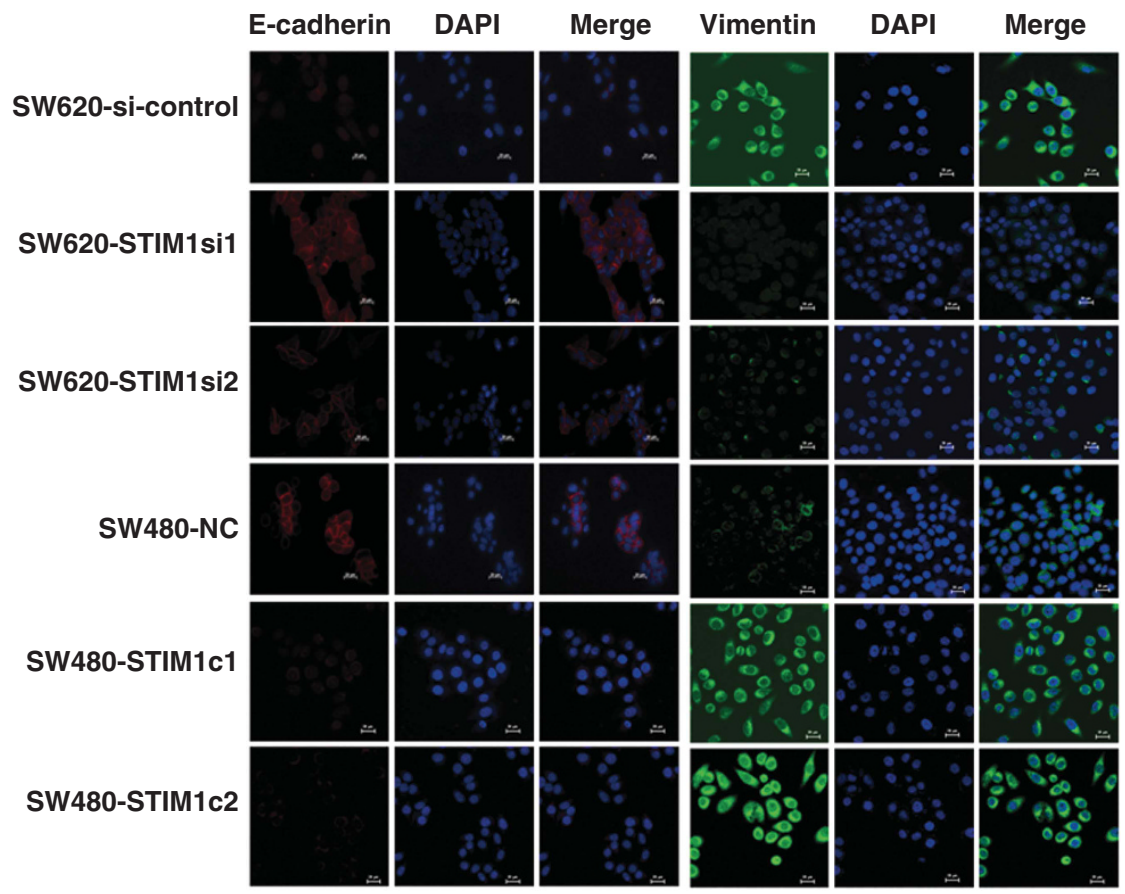

Figure 3. STIM1 regulates EMT in CRC cells. (a) Morphological changes by STIM1 in SW620 and SW480 cells. Scale bar, $30 \mu \mathrm{m}$. (b-d) Western blotting, real-time PCR and IF staining show upregulated expression of epithelial markers and downregulated expression of mesenchymal markers in SW620-STIM1si1 and SW620-STIM1si2 cells. In contrast, overexpression of of STIM1 resulted in decreased expression of epithelial markers and increased expression of mesenchymal markers in SW480 cells. Scale bar, $20 \mu \mathrm{m} .{ }^{*} P<0.05$. 
the metastatic phenotype of CRC cells, SW620 cells were transfected with STIM1-specific small interfering RNAs (siRNAs) or a control siRNA. Three different siRNAs targeting STIM1 were tested, and the two that most effectively knocked down STIM1 expression (SW620-STIM1si1 and SW620-STIM1si2) were selected for subsequent studies. SW480 cells were infected with recombinant lentivirus expressing STIM1 (LV-STIM1) or a control lentivirus (LV-NC) and stable clones were established (SW480-STIM1C1, SW480-STIM1C2 and SW480-NC, respectively; Figure 1b). Upregulation and knockdown of STIM1 expression was confirmed by western blot analysis (Figure 1b). Transwell assays showed that downregulation of STIM1 expression in SW620 cells markedly decreased cell migration and invasion (Figures 1c1 and c2). Conversely, upregulation of STIM1 expression in SW480 cells significantly enhanced cell migration and invasion (Figures 1d1 and d2). MTT (3-(4,5-dimethythiazol-2-yl)-2,5diphenyl tetrazolium bromide) assay was performed to exclude the contribution of cell proliferation (Supplementary Figures S1A and $\mathrm{B})$.These results suggest that STIM1 promotes metastasis in CRC cells.

To further explore the role of STIM1, we injected SW480STIM1c1, SW480-STIM1C2, SW620-STIM1si1 or SW620-STIM1si2 cells into nude mice through the lateral tail vein. Histologic analyses (Figures $2 \mathrm{c} 1$ and $\mathrm{c} 2$ ) confirmed that the incidence of lung and liver metastases was markedly increased in mice injected with SW480-STIM1C1 or SW480-STIM1C2 cells and markedly decreased in mice injected with SW620-STIM1si1 or SW620-STIM1si2 cells, compared with the control group. The number of metastatic lung
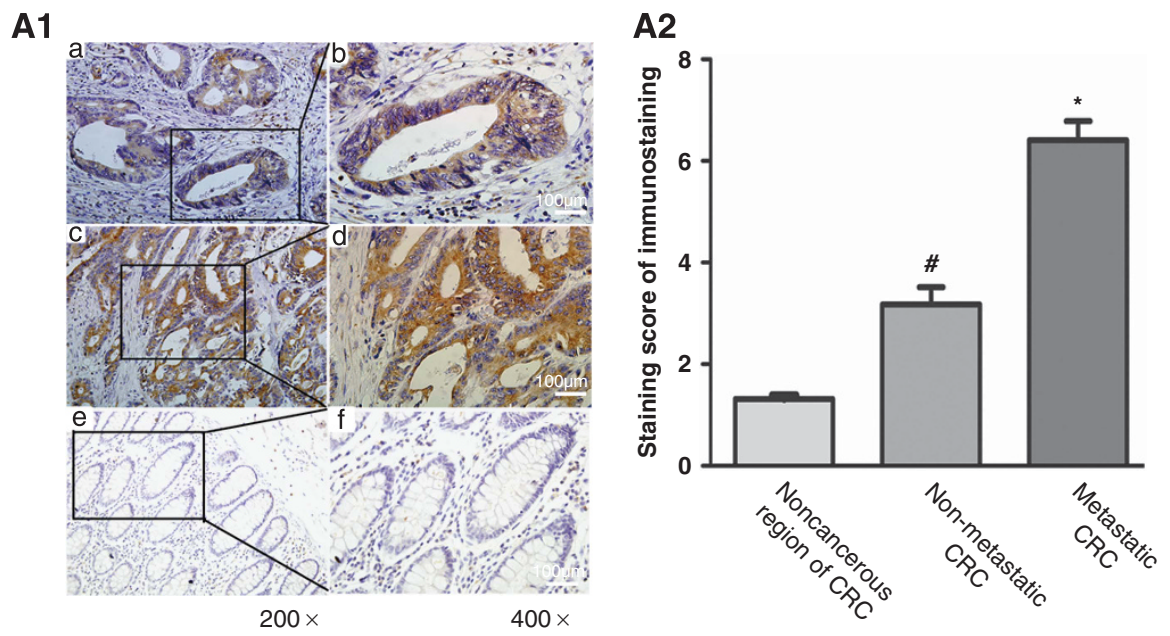

A3
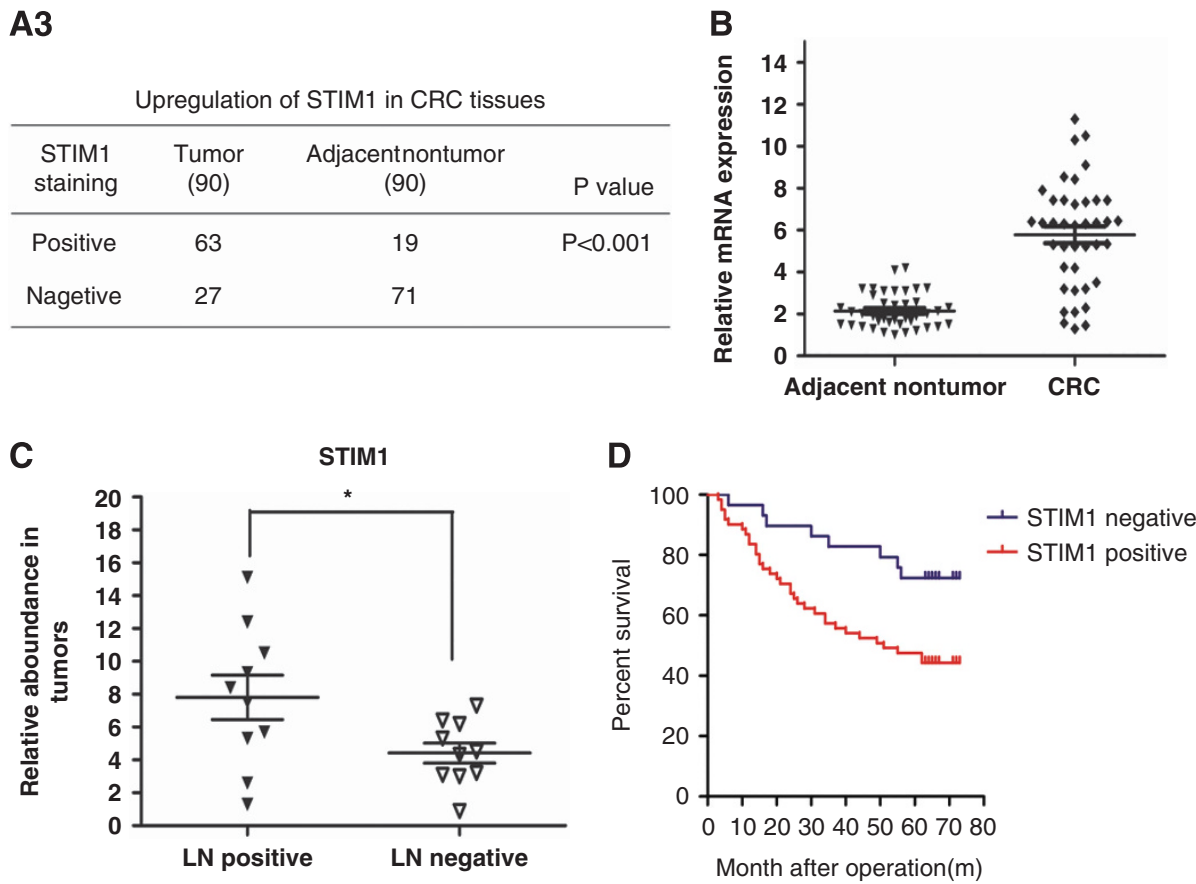

Figure 4. STIM1 is significantly upregulated in CRC tissues. (A1) Immunohistochemistry analysis of STIM1 expression in 90 paired CRC tissues. (a, c and e) Magnification, $\times 200$; (b, d and $\mathbf{f}$ ) magnification, $\times 400$. (a and b) Primary sites of non-metastatic CRC; (c and d) primary sites of metastatic CRC; and (e and f) noncancerous region of CRC. Scale bar, $100 \mu \mathrm{m}$. (A2) Results of immunohistochemical staining were evaluated by the staining scores. ${ }^{*} P<0.05$ vs non-metastatic CRC or noncancerous region of CRC. ${ }^{\#} P<0.05$ vs noncancerous region of $C R C$. (A3) Statistical analysis of STIM1 expression in CRCs. (B) Real-time PCR analysis of STIM1 expression in 40 pairs of CRC and adjacent nontumorous tissues. (C) Real-time qPCR analysis of STIM1 abundance in human CRC primary tumors, LN-positive $(n=10)$ or -negative $(n=10)$. Matching normal colorectal tissue sample from the same patient was used for normalization. (D) Kaplan-Meier's analysis of the correlation between STIM1 expression and overall survival (OS) of CRC patients. 
and liver nodules was significantly increased in the SW480STIM1C1 and SW480-STIM1C2 groups and significantly decreased in the SW620-STIM1si1 and SW620-STIM1si2 groups, compared with the control group (Figures 2a, b1 and b2).

\section{STIM1 regulates EMT in CRC cells}

The EMT has a crucial role in cancer metastasis. During the EMT process, differentiated polarized epithelial cells undergo profound morphogenetic changes, typified by the dissolution of cell-cell junctions and loss of apicobasolateral polarity, resulting in the formation of migratory mesenchymal cells with invasive properties. Moreover, during this process the expression of epithelial markers (such as E-cadherin and $\beta$-catenin) is downregulated, whereas the expression of mesenchymal markers (such as vimentin and fibronectin) is upregulated. ${ }^{38,39}$

By comparing the morphology of the cell models described above under a light microscope, we observed that ectopic expression of STIM1 in SW480 cells induced the conversion of polarized epithelial cells to spindle-shaped, fibroblast-like mesenchymal cells with the loss of cell-cell adhesion, whereas knockdown of STIM1 in SW620 cells resulted in a tighter organization of cells in colonies and an epithelioid morphology (Figure 3a).

Next, we assessed the expression of EMT markers in these cell models. Western blotting and quantitative reverse transcriptionPCR (qRT-PCR) analysis showed a marked decrease in the expression of vimentin and fibronectin and a significant increase in the expression of E-cadherin and $\beta$-catenin in SW620-STIM1si1 and SW620-STIM1si1 cells, compared with control cells. Conversely, a decrease in the expression of E-cadherin and $\beta$-catenin and an increase in the expression of vimentin and fibronectin were observed in SW480-STIM1C1 and SW480-STIM1c1 cells, compared with control cells (Figures $3 \mathrm{~b}$ and c). These changes in E-cadherin and vimentin expression were confirmed by an immunofluorescent assay (Figure $3 \mathrm{~d}$ ). These results suggest that STIM1 is critical for the acquisition of EMT characteristics and may contribute to the EMT-derived invasive phenotype in CRC cells.

Table 1. Correlation between STIM1 expression and clinicopathological characteristics of 90 CRC

\begin{tabular}{|c|c|c|c|c|}
\hline \multirow[t]{2}{*}{ Variable } & \multirow[t]{2}{*}{$\begin{array}{l}\text { Number of } \\
\text { cases }\end{array}$} & \multicolumn{2}{|c|}{$\begin{array}{c}\text { STIM1 } \\
\text { immunostaining }\end{array}$} & \multirow[t]{2}{*}{ P-value } \\
\hline & & - & + & \\
\hline \multicolumn{5}{|l|}{ Age (years) } \\
\hline$\geqslant 60$ & 66 (73.3\%) & 18 & 48 & \\
\hline$<60$ & $24(26.7 \%)$ & 9 & 15 & 0.436 \\
\hline \multicolumn{5}{|l|}{ Gender } \\
\hline Male & 47 (52.2\%) & 15 & 32 & \\
\hline Female & $43(47.8 \%)$ & 12 & 31 & 0.818 \\
\hline \multicolumn{5}{|l|}{ Tumor size $(\mathrm{cm})$} \\
\hline$\geqslant 5$ & $54(55.6 \%)$ & 12 & 42 & \\
\hline$<5$ & $36(44.4 \%)$ & 15 & 21 & 0.062 \\
\hline \multicolumn{5}{|l|}{ TNM stage } \\
\hline Stage $1 / I I$ & 56 (62.2\%) & 24 & 32 & \\
\hline Stage III/IV & $34(27.8 \%)$ & 3 & 31 & 0.001 \\
\hline \multicolumn{5}{|l|}{ Lymph node status } \\
\hline No metastasis & 57 (63.3\%) & 22 & 35 & \\
\hline Metastasis & 33 (36.7\%) & 5 & 28 & 0.031 \\
\hline
\end{tabular}

STIM1 is significantly upregulated in human CRC tissues and indicates poor prognosis

To explore the potential role of STIM1 in determining the clinical outcome of CRC, we assessed STIM1 expression in a tissue microarray of 90 CRC patients. The results of immunohistochemical staining showed that STIM1 was localized to both the cytoplasm and membranes. Positive STIM1 expression was detected in 63 of 90 (71.1\%) primary CRC samples compared with only 19 of 90 (21.1\%) adjacent non-tumor tissues. These results were confirmed by scoring the staining intensity across the section (Figures 4A1-3). These findings indicated that STIM1 was significantly overexpressed in human CRC tissues. To investigate the correlation between STIM1 overexpression and CRC metastasis, we compared STIM1 expression in 10 pairs of LN metastasis-positive (LN-positive group) and LN metastasisnegative (LN-negative group) primary CRC specimens. Real-time PCR analysis showed that STIM1 mRNA levels were much higher in the LN-positive group than in the LN-negative group (Figure 4C). Furthermore, Kaplan-Meier analysis showed that patients with positive STIM1 expression had shorter overall survival than those who were negative for STIM1 expression (Figure 4D). To investigate whether STIM1 expression is associated with the progression of CRC, we investigated the expression of STIM1 in an additional 40 fresh CRC tissues by qRT-PCR (Figure 4B) and compared the results with the clinicopathologic characteristics of the patients. The results showed that overexpression of STIM1 did not correlate with age, gender or tumor size $(P>0.05)$, but was significantly associated with TNM stage and LN status $(P<0.01$; Table 1). Taken together, these results suggest that the expression level of STIM1 positively correlated with the progression of CRC and that STIM1 overexpression is indicative of poor prognoses of human CRC patients following curative resection.

The expression of miR-185 inversely correlates with the expression of STIM1 in CRC cells and is associated with the progression of CRC The above results indicated that STIM1 overexpression might have a critical role in CRC. However, there have been few studies on the regulation of STIM1 expression in cancer. miRNAs were recently found to have an important role in tumor progression through their function as posttranscriptional regulators. We hypothesized that posttranscriptional regulation by miRNAs may represent one of the upstream regulatory mechanisms of STIM1 expression. To test this idea, we used several web-based target analysis tools (TargetScan, miRanda, RNAhybrid, pictar and DIANAmT) to identify miRNAs that potentially target STIM1. As a result, miR-185 was identified as a potential regulator of STIM1 expression (Figure 5a). This was consistent with the results of our previous miRNA microarray analysis, which identified miR-185 as the most significantly downregulated miRNA in SW620 cells compared with SW480 cells. ${ }^{36}$ Therefore, miR-185 was selected for further study.

We examined endogenous miR-185 expression levels in four human CRC cell lines (SW480, SW620, HT29 and LOVO) and in a normal human intestinal epithelial cell line by qRT-PCR. The expression levels of miR-185 were significantly decreased in SW620 and LOVO cell lines (highly metastatic cells) compared with either poorly metastatic or immortalized cells. miR-185 expression negatively correlated with the metastatic potential of the CRC cells (Figure 5b1). Furthermore, the expression of miR-185 and STIM1 was inversely correlated (Figure $5 \mathrm{~b} 2$ ). To investigate the correlation of miR-185 with STIM1 expression levels in CRC tissues, we used qRT-PCR to measure the expression levels of miR-185 in the 40 CRC tissues previously described. Our results confirmed that the mRNA level of miR-185 negatively correlated with STIM1 expression (Figure $5 \mathrm{c} 2$ ). The correlation of miR-185 expression with clinicopathologic factors was also examined. In contrast to STIM1 expression, low expression levels of miR-185 were significantly associated with advanced clinical tumor node 
a

\begin{tabular}{|c|c|c|}
\hline $\begin{array}{r}3, \\
38: 5^{\prime}\end{array}$ & $\begin{array}{c}\text { agucCUUGACGGAAAGAGAGGu } \\
\text { I : : : : I I I I I I I I I } \\
\text { cccuGGGUGUÚCUGUCUCUCCu }\end{array}$ & $\begin{array}{l}\text { hsa-miR-185 } \\
\text { STIM1 }\end{array}$ \\
\hline
\end{tabular}

b1

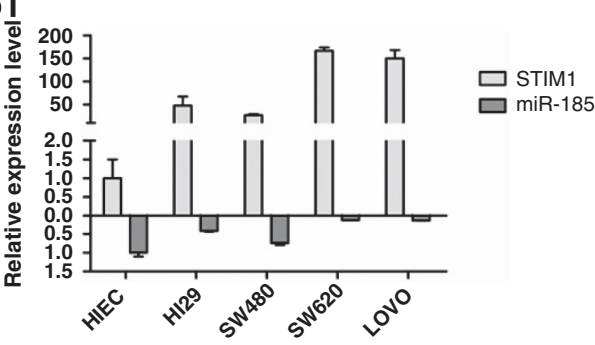

c1

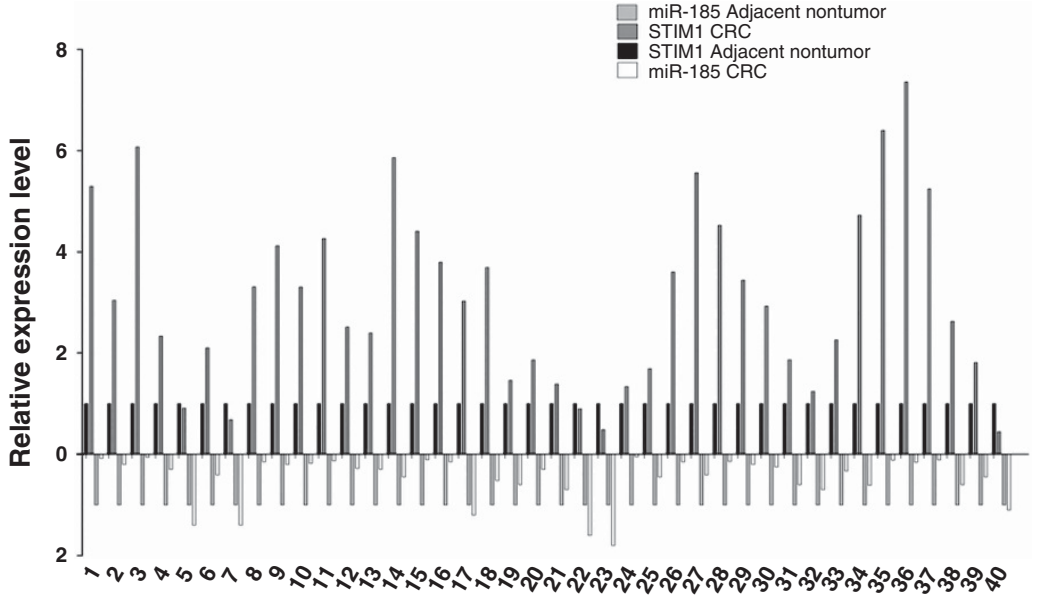

b2

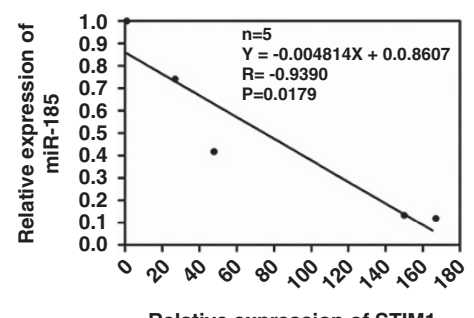

Relative expression of STIM1

-185 Adjacent nontumo STIM1 CRC

(18) nontumor

c2

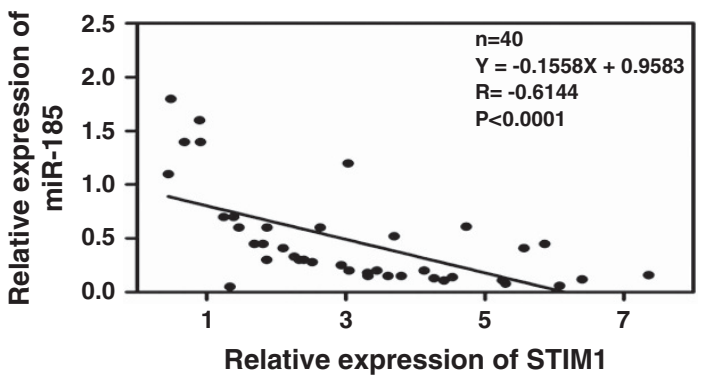

Figure 5. Expression of miR-185 and STM1 negatively correlates in CRC cell lines and clinical samples. (a) Sequence alignment of miR-185 with binding site on the STIM1 3'-UTR. (b1) Expression of miR-185 and STIM1 in CRC cells was analyzed by qPCR. (b2) Scatter plots showing the negative linear correlation between the mRNA expression of miR-185 and that of STIM1 in CRC cells. (c1) qPCR analysis of the expression of miR-185 and STIM1 in CRC tissues. (c2) Scatter plots showing the negative linear correlation between the mRNA expression of miR-185 and that of STIM1 in CRC tissues.

metastasis stage and LN metastasis $(P<0.001)$. miR-185 expression levels in CRC patients did not correlate with age, gender, tumor size or cell differentiation $(P>0.05)$ (Table 2$)$. These results strongly suggest that miR-185 is one of the upstream molecules regulating STIM1 expression in cancer.

miR-185 represses invasion and regulates EMT in CRC cells

To investigate the role that miR-185 has in the migration and invasion of CRC cells, the SW620 cell line was transiently transfected with miR-185 mimics. Successful upregulation of miR-185 expression was confirmed by qRT-PCR (Figure 6a1). Increased miR-185 expression resulted in significant inhibition of migration and invasion in SW620 cells (Figures 6a2 and a3). To determine whether the loss of miR-185 promotes the migration or invasion of CRC cells, miR-185 was silenced in SW480 cells with an antisense oligonucleotide inhibitor (Figure 6b1). This inhibition of miR-185 significantly promoted cell migration and invasion (Figures $6 \mathrm{~b} 2$ and b3). These results indicate that miR-185 represses the invasive potential of CRC cells. As STIM1 has been shown to promote EMT in CRC cells and miR-185 has been predicted as a potential regulator of STIM1, we hypothesized that miR-185 may exert an opposite effect on the regulation of EMT in CRC cells. To test this, we compared the morphology of our cell models under a light microscope, and found that upregulating the expression of miR-185 in SW620 cells induced a tighter organization of cells in colonies, whereas downregulating the expression of miR-185 in SW480 cells led to a more fibroblast-like mesenchymal cell morphology (Figure 3c). Western blot and qRT-PCR analyses showed that upregulation of miR-185 in SW620 cells increased the 
Table 2. Clinicopathological associations of miR-185 expression in primary CRC

\begin{tabular}{|c|c|c|c|}
\hline Variable & $\begin{array}{l}\text { Number of } \\
\text { cases }\end{array}$ & $\begin{array}{c}\text { Median expression of } \\
\text { miR-185 (range) } \\
(2-\Delta \Delta C T)^{*}\end{array}$ & P-value \\
\hline \multicolumn{4}{|l|}{ Age (years) } \\
\hline$\geqslant 60$ & 21 (52.5\%) & $3.7(1.7-13.1)$ & \multirow[t]{2}{*}{0.536} \\
\hline$<60$ & $19(47.5 \%)$ & $3.6(1.0-28.2)$ & \\
\hline \multicolumn{4}{|l|}{ Gender } \\
\hline Male & $23(57.5 \%)$ & $3.6(1.2-28.2)$ & \multirow{2}{*}{0.962} \\
\hline Female & $17(42.5 \%)$ & $3.4(1.0-13.1)$ & \\
\hline \multicolumn{4}{|l|}{ Tumor size $(\mathrm{cm})$} \\
\hline$\geqslant 5$ & 23 (57.5\%) & $4.2(1.2-28.2)$ & \multirow[t]{2}{*}{0.554} \\
\hline$<5$ & $17(42.5 \%)$ & $3.4(1.0-11.4)$ & \\
\hline \multicolumn{4}{|c|}{ Degree of differentiation } \\
\hline $\begin{array}{l}\text { Well and } \\
\text { moderately }\end{array}$ & $20(50.0 \%)$ & $4.2(1.2-28.2)$ & \multirow[t]{2}{*}{0.113} \\
\hline $\begin{array}{l}\text { Poorly } \\
\text { differentiated }\end{array}$ & $20(50.0 \%)$ & $3.1(1.0-20.4)$ & \\
\hline \multicolumn{4}{|l|}{ TNM stage } \\
\hline Stage I/II & 25 (62.5\%) & $4.6(1.2-28.2)$ & \multirow{2}{*}{0.041} \\
\hline Stage III/IV & 15 (37.5\%) & $3.2(1.0-10.3)$ & \\
\hline \multicolumn{4}{|l|}{ Lymph node status } \\
\hline No metastasis & 21 (52.5\%) & $4.7(1.2-28.2)$ & \multirow[t]{2}{*}{0.011} \\
\hline Metastasis & $19(47.5 \%)$ & $3.2(1.0-11.4)$ & \\
\hline
\end{tabular}

expression of E-cadherin and $\beta$-catenin and decreased the expression of vimentin and fibronectin (Figures $6 \mathrm{~d}$ and e1, 2). Moreover, inhibition of miR-185 in SW480 cells decreased the expression of E-cadherin and $\beta$-catenin and increased the expression of vimentin and fibronectin (Figures $6 \mathrm{c}$ and $\mathrm{d} 2$ ). Interestingly, these effects of miR-185 on EMT were similar to the effects of STIM1 siRNAs in CRC cells.

STIM1 is a direct functional target of miR-185 in CRC metastasis As mentioned above, an inverse correlation between miR-185 and STIM1 expression was identified in both CRC cells and tissues. To obtain additional direct evidence that STIM1 expression is regulated by miR-185, we analyzed the binding site of miR-185 in the $3^{\prime}$-untranslated region (UTR) of STIM1 and performed a luciferase reporter assay. An STIM1 3'-UTR reporter (Luc-STIM1) or the corresponding mutant reporter (Luc-STIM1-mu) was cotransfected with miR-185 mimics or mimic controls into SW620 cells. As expected, the luciferase activities of the reporter constructs were reduced significantly only when miR-185 mimics were co-transfected with Luc-STIM1 in SW620 cells. No apparent change in luciferase activities was observed in cells transfected with Luc-STIM1-mu or mimic controls (Figure 7a). To confirm whether targeting of the STIM1 3'-UTR by miR-185 was functional, SW620 cells transfected with miR-185 mimics and SW480 cells transfected with the miR-185 inhibitor were analyzed by qRT-PCR and western blotting. Both mRNA (Figure 7b1) and protein (Figure 7b2) levels of STIM1 were markedly upregulated in SW480 cells transfected with the miR-185 inhibitor, whereas both mRNA (Figure $7 c 1$ ) and protein (Figure $7 c 2$ ) levels of STIM1 were effectively downregulated in SW620 cells transfected with the miR-185 mimic. Further experiments confirmed that the altered miR-185 expression does not affect the expression of STIM2, Orai1, Orai2 and Orai3, which may also be involved in SOCE
(Supplementary Figures S2A and B). These results suggest that STIM1 is a direct functional target of miR-185. To further corroborate this hypothesis, we performed a 'rescue' experiment by co-transfecting SW620 cells with miR-185 mimics and a pcDNA3.1 vector carrying an STIM1 expression cassette (pcDNA3.1-STIM1) or mutated seed sequences of miR-185 at the STIM1 3'-UTR (STIM1-mu). Western blot analysis demonstrated that co-transfection of SW620 cells with the miR-185 mimic and STIM1-mu restored STIM1 protein expression, whereas COtransfection with pCDNA3.1-STIM1 failed to recover the STIM1 expression that was silenced by the miR-185 mimic. Furthermore, restoration of STIM1 expression by the introduction of STIM1-mu into SW620 cells transfected with the miR-185 mimics inhibited the expression of E-cadherin and $\beta$-catenin and increased the expression of vimentin and fibronectin (Figure 7d). In addition, rescuing STIM1 expression in the presence of the miR-185 mimics enhanced the invasion and migration potential of SW620 cells (Figure 7e). Moreover, to determine the effect of the miR-185STIM1 axis on SOCE, we examined cellular $\mathrm{Ca}^{2+}$ distribution by $\mathrm{Ca}^{2+}$ imaging in both SW620 and SW480 cells. After ER Ca ${ }^{2+}$ depletion, $\mathrm{Ca}^{2+}$ influx through SOCE into SW620 cells was increased compared with SW480 cells (Supplementary Figures $\mathrm{S} 3 \mathrm{~A}$ and $\mathrm{B})$. We then examined the effect of overexpression of miR-185 and found that SOCE was impaired in SW620-miR-185 mimic cells (Supplementary Figures S3C and D), whereas inhibition of miR-185 in SW480 cells moderately increased SOCE (Supplementary Figures S3E and F). Taken together, these results suggest that STIM1 is a potential functional target of miR-185.

In summary, our results suggest that STIM1 is a direct functional target of miR-185 and that this novel miR-185/STIM1 axis has an important role in the regulation of CRC metastasis.

\section{DISCUSSION}

In this study, we demonstrate that STIM1 promotes CRC metastasis. The upregulation of STIM1 in CRC is at least partly attributed to posttranscriptional regulation by miR-185, a novel EMT-regulating miRNA. Additionally, we provide evidence supporting the involvement of STIM1 in the regulation of EMT in cancer.

The influx of $\mathrm{Ca}^{2+}$ is essential for the migration of tumor cells. ${ }^{40,41}$ STIM1, an endoplasmic reticulum $\mathrm{Ca}^{2+}$ sensor, regulates $\mathrm{Ca}^{2+}$ influx by activating SOCE. ${ }^{11,12}$ STIM1 has recently been proposed to be a critical molecule in the regulation of cancer metastasis, ${ }^{21,22}$ although the exact role of STIM1 in metastasis remains controversial. STIM1 was initially implicated as an antimetastasis molecule. ${ }^{19}$ However, we found that STIM1 promoted migration and invasive properties in CRC, and that increased expression of STIM1 in CRC was significantly associated with cancer metastasis. In accordance with our study, STIM1 was reported to be one of the molecules essential for breast tumor cell migration in vitro and tumor metastasis in vivo. ${ }^{21}$ Similarly, another study demonstrated that the level of STIM1 was significantly associated with the risk of metastasis in cervical cancer. Moreover, epidermal growth factor-stimulated migration in cervical cancer cells requires STIM1 expression. ${ }^{22}$ Our results provide new evidence supporting the involvement of STIM1 in the promotion of cancer metastasis.

It has been reported that expression of constitutively active Ras overcomes the migration-inhibitory function of the $\mathrm{Ca}^{2+}$ influx inhibitor and STIM1 SiRNA in breast cancer. ${ }^{21}$ Interestingly, a previous study has shown that activation of the Ras signaling pathway leads to activation of the transcriptional regulator, Snail1, ${ }^{28}$ which appears to have a major role in EMT. ${ }^{23,29}$ These studies strongly suggest that STIM1 might promote cancer metastasis by regulating the EMT. In this study, we demonstrated that knockdown of STIM1 induced the conversion of spindle- 

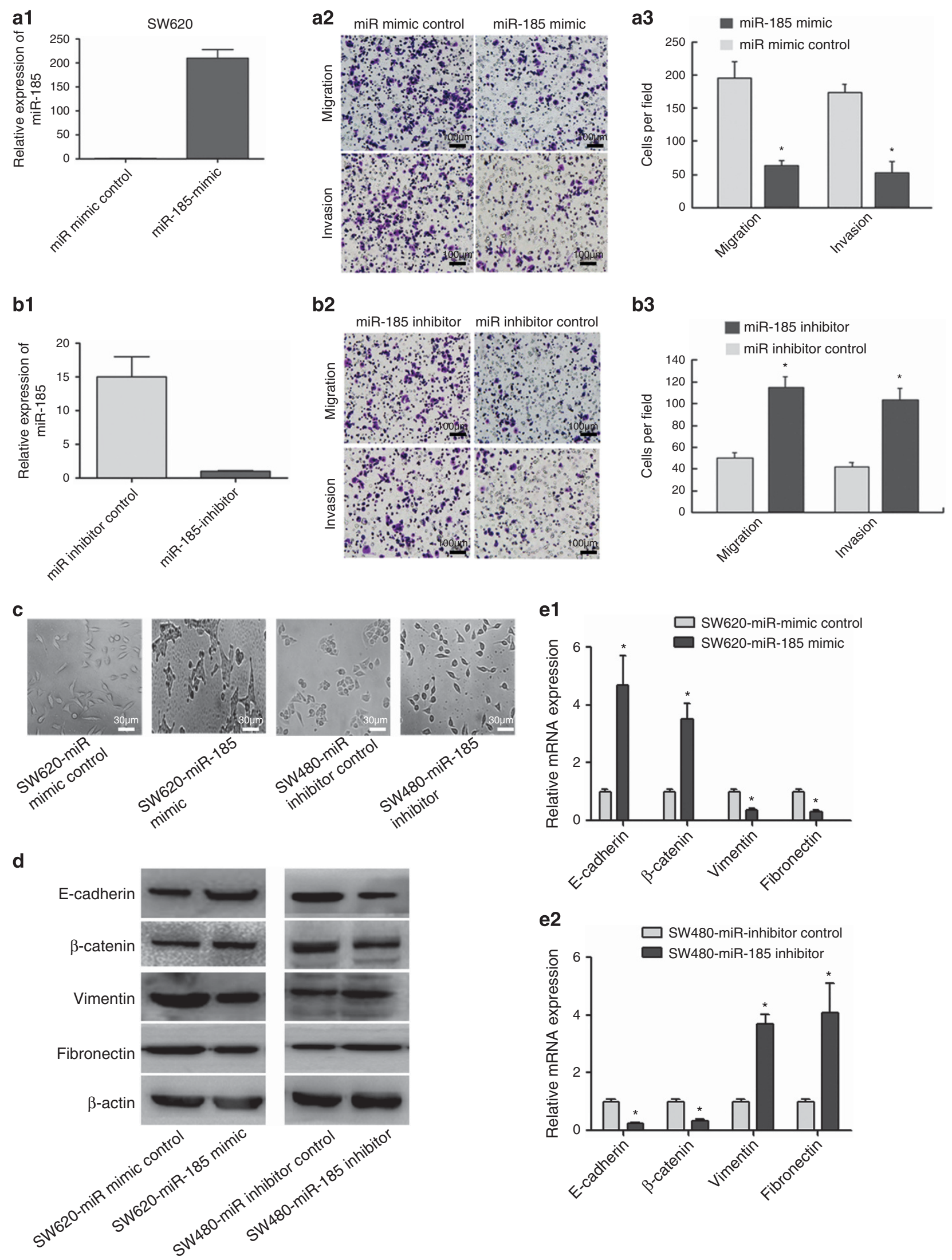
shaped, fibroblast-like mesenchymal cells to polarized epithelial cells, enhanced invasion and migration in CRC cells, upregulated epithelial markers and downregulated mesenchymal markers. These findings provided new evidence supporting the involvement of STIM1 in driving metastasis through the regulation of EMT in cancer cells.

We further examined the clinical relevance of STIM1 and found that STIM1 was markedly upregulated in CRC tissues. Moreover, increased levels of STIM1 correlated with a high metastatic potential of CRC cells. In accordance with our findings, a previous study also showed that STIM1 was significantly associated with the risk of metastasis in cervical cancer. ${ }^{22}$ Based on these findings, elucidating the mechanism of STIM1 upregulation may contribute to the identification of new cancer therapy targets. A recent study demonstrated that the expression of STIM1 is regulated by Wilms tumor suppressor 1 and early growth response 1 in six specific cancer subtypes. ${ }^{42}$ Another study reported that STIM1 is regulated by androgens in prostate stromal cells. However, these observations are not sufficient to explain the mechanism behind the upregulation of STIM1 in cancer. A recent study found that another STIM protein, STIM2, inversely control melanoma growth and invasion, ${ }^{43}$ suggesting that STIM proteins, including STIM1 and STIM2, have critical roles in cancer. Interestingly, these STIM proteins may exert different and even opposite functions in different kinds of cancers, which imply complex and important roles in cancer. Further study on STIM family may provide new targets for cancer therapy.

miRNAs have been implicated in the regulation of tumor metastasis ${ }^{32}$ and recent studies have highlighted the importance of miRNAs in the regulation of the EMT. ${ }^{33,34}$ To explore whether posttranscriptional regulation by certain miRNAs is one of the upstream regulatory mechanisms of STIM1 expression, we used several target prediction algorithms to assay for miRNA binding sites in the STIM1 3'-UTR. miRNA-185 was identified by several algorithms. Surprisingly, our previous miRNA microarray analysis revealed that miR-185 was the most significantly downregulated miRNA in SW620 cells (highly metastatic) compared with SW480 cells (poorly metastatic). ${ }^{36}$ In this study, we found that miR-185 expression inversely correlated with the expression of STIM1 in both CRC cells and tissues, and that transfection with miR-185 significantly downregulated the expression of STIM1. These results were further confirmed by luciferase activity assays. miR-185 was recently found to be an independent prognostic factor in gastric cancer $^{44}$ and hepatocellular carcinoma. ${ }^{45}$ Moreover, miR-185 can induce cell cycle arrest and repress cell proliferation in non-smallcell lung cancer, ${ }^{46}$ colorectal cancer, ${ }^{47}$ glioma, ${ }^{48}$ gastric cancer ${ }^{49}$ and breast cancer. ${ }^{50}$ miR-185 has also been reported to be involved in metastasis in colorectal cancer $^{51}$ and glioma. ${ }^{48}$ However, the role of miR-185 in the pathophysiological functions of STIM1 has not previously been reported.

The results presented here show that upregulating the expression of miR-185 inhibited migration and invasion and reversed the EMT in CRC cells. Our data further indicate that STIM1 is a functional target of miR-185 in CRC. Taken together, these findings provide new insight into the mechanism of the regulation of STIM1 expression at the posttranscriptional level, although further studies are required to fully understand the mechanism by which the miR-185-STIM1 pathway regulates EMT in cancer.

In conclusion, we have demonstrated a critical function of STIM1 in promoting CRC metastasis. This study also reveals a new regulatory mechanism of STIM1 expression by miR-185, an miRNA not previously associated with the EMT process. We believe that the miR-185-STIM1 pathway is an attractive target for therapeutic intervention against cancer metastasis.

\section{MATERIALS AND METHODS}

\section{Cell culture}

The human CRC cell lines were preserved in our institute and were routinely maintained in RPMI-1640 medium (HyClone, Logan, UT, USA) supplemented with $10 \%$ fetal bovine serum (Gibco, Carlsbad, CA, USA), $100 \mathrm{U} / \mathrm{ml}$ penicillin sodium and $100 \mathrm{mg} / \mathrm{ml}$ streptomycin sulfate at $37^{\circ} \mathrm{C}$ in a humidified air atmosphere containing 5\% carbon dioxide. Throughout the experiment, cells were used in the logarithmic phase of growth according to the supplier's instructions.

\section{Clinical samples}

Samples from 40 CRC patients (23 males and 17 females) who had undergone proctocolectomy with $\mathrm{LN}$ dissection for CRC at Xijing Hospital (Xi'an, China) between November 2008 and April 2009 were included in the study (Supplementary Table S2). None of the patients received preoperative chemotherapy. The resected specimens were histologically examined by hematoxylin and eosin staining. The primary tumor samples and corresponding non-tumor mucosa were collected from each patient immediately after the surgical process and were snap frozen in liquid nitrogen until further use. Total RNA from the frozen tissues was isolated with TRIzol (Invitrogen, Carlsbad, CA, USA) according to the manufacturer's instructions.

Oligonucleotide construction, lentivirus production and construct design

An miR-185 mimic, an inhibitor and a negative control oligonucleotides were purchased from RiboBio (Guangzhou, China). Transfection of oligonucleotides was performed using Lipofectamine 2000 (Invitrogen) according to the manufacturer's instructions. On the basis of the STIM1 sequence, three short hairpin RNAs were designed using the siRNA Target Finder (InvivoGene, San Diego, CA, USA): 5'-GGGAAGACCTCAATTACCA-3', 5'-TCTCCACATTTGGATTCTT-5' and 5'-TGGATGATGTAGATCATAA- $3^{\prime}$. Lentiviral vectors encoding short haipin RNAs were generated using GV248 vector (GeneChem Co., Ltd, Shanghai, China). Stable transfectants overexpressing STIM1 were generated by lentiviral transduction using a GV166 vector (GeneChem Co., Ltd). An empty vector was used as the negative control.

STIM1-expressing vector with miR-185 binding sites or mutated seed sequences of miR-185 at STIM1 3'-UTR (STIM1-mu) was purchased from Open Biosystems (Huntsville, AL, USA) and was subcloned into the eukaryotic expression vector.

\section{Luciferase assay}

Luc-STIM1 vector. The STIM1 3'-UTR containing the predicted miR-185 binding site was amplified by RT-PCR from the total RNA of cultured

Figure 6. miR-185 represses the migration and invasion potential and reverses the EMT phenotype in CRC cells. (a1) qRT-PCR analysis of miR-185 in SW620 cells transfected with an miR-185 mimic or a negative control. (a2) Invasion and migration assay of SW620 cells transfected with an miR-185 mimic or a negative control. Representative fields of migration (top) or invasive (bottom) cells on membrane Scale bar, $100 \mu \mathrm{m}$. (a3) Average number of invasive or migration cells number per field from three independent experiments \pm s.e. ${ }^{*} P<0.05$. (b1) qRTPCR analysis of miR-185 in SW480 cells transfected with an miR-185 inhibitor or a negative control. (b2) Invasion or migration assay of SW480 cells transfected with an miR-185 inhibitor or a negative control. Scale bar, $100 \mu \mathrm{m}$. (b3) Average number of invasive or migration cells number per field from three independent experiments \pm s.e. ${ }^{*} P<0.05$. (c) Morphological changes by miR-185 in SW620 and SW480 cells. Scale bar, $30 \mu \mathrm{m}$. (d) Expression of EMT markers was determined by western blotting in SW620 cells transfected with an miR-185 mimic or a negative control and SW480 cells transfected with an miR-185 inhibitor or a negative control. (e1 and e2) Expression of EMT markers was determined by qRT-PCR in the above four cell lines. ${ }^{*} P<0.05$. 
a

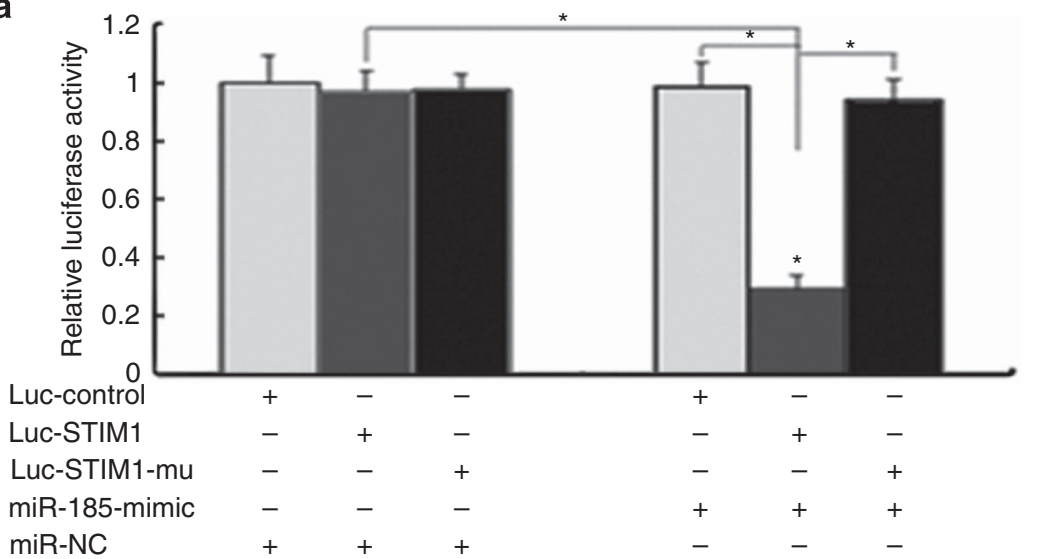

b2

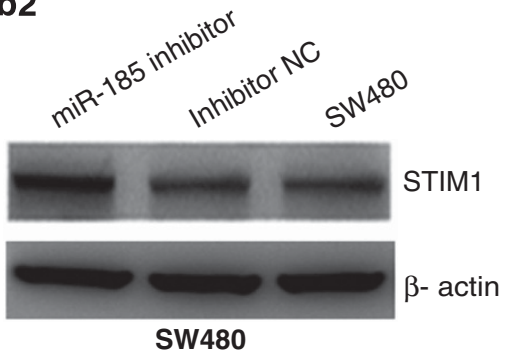

c1

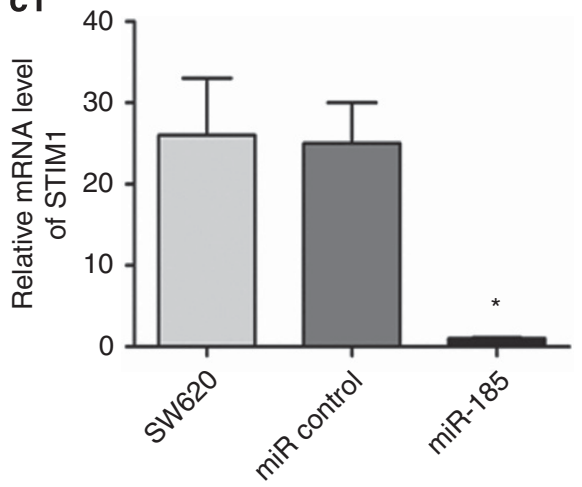

b1

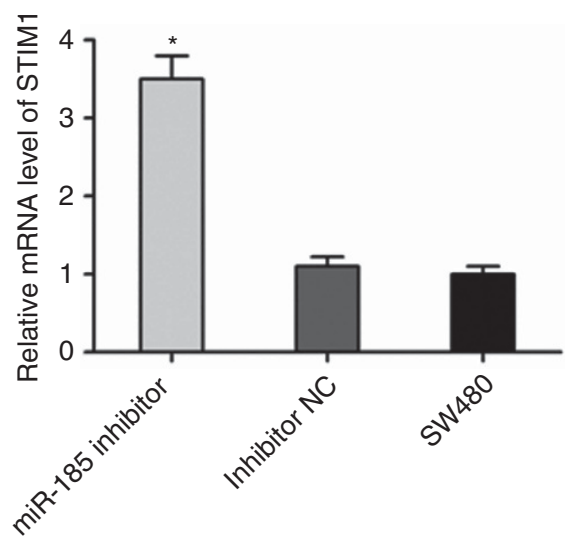

c2

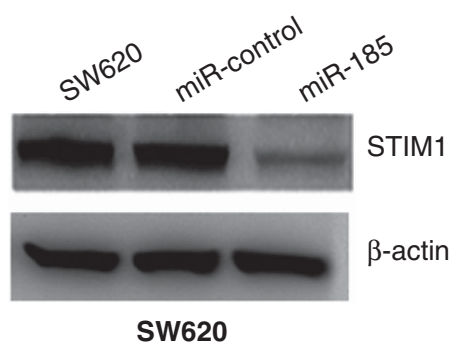

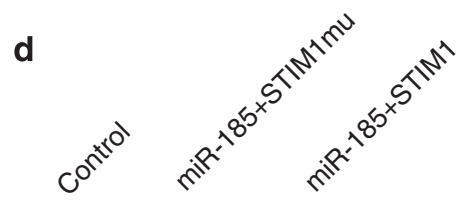

e
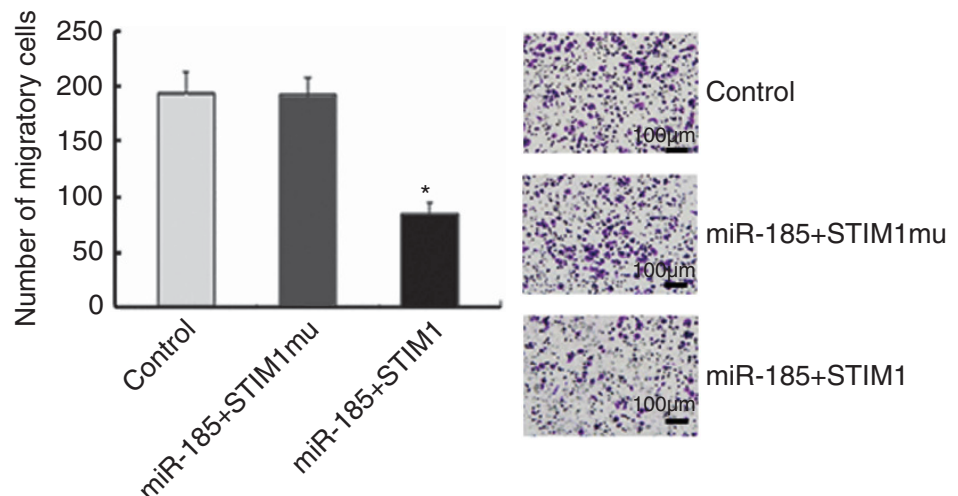

Figure 7. Directly targeting of STIM1 by miR-185 in CRC EMT. (a) Luciferase activity assay for targeting of the 3'-UTR of STIM1 by miR-185. The wild-type and mutant miR-185 target sequences of STIM1 were fused with luciferase reporter and transfected into vector control (Luc-STIM1 and Luc-STIM1-mu). Luc-STIM1, Luc-STIM1-mu or the vector control was co-transfected with miR-185 or an miRNA negative control in SW620 cells, and the luciferase activity measured. $P<0.05$. (b1 and c1) STIM1 mRNA levels were analyzed by qRT-PCR. (b2 and c2) STIM1 protein levels were analyzed by western blotting. $\beta$-Actin was used as an internal control. (d) Expression of STIM1 and EMT markers was determined by western blotting in SW620 cells. MiR-185 was co-transfected with an STIM1 wild-type construct (STIM1) or an STIM1 mutant construct in which the target sequence of miR-185 was mutant (STIM1-mu) in SW620 cells. $\beta$-Actin was used as an internal control. (e) Migration assay of SW620 cells. SW620 cells were treated as in (d). Average number of invasive or migration cell number per field from three independent experiments \pm s.e. is at the right. ${ }^{*} P<0.05$ (left). Representative fields of migration (top) or invasive (bottom) cells on the membrane is at the right. Scale bar, $100 \mu \mathrm{m}$.

SW620 cells and was cloned into the pCR2.1-TOPO vector (Invitrogen). The pCR2.1-TOPO-STIM1-3'-UTR construct was digested with Spel and HindIII. The resulting fragment was subcloned into the Spel and Hindlll sites of the pMIR-REPORT miRNA expression reporter vector (Applied Biosystems, Foster City, CA, USA). The first six nucleotides complementary to the miR-185 seed region were deleted from the mutant constructs using the
QuikChange Site-Directed Mutagenesis Kit (Stratagene, La Jolla, CA, USA) according to the manufacturer's protocol. Standard 3'-UTR luciferase assays were performed to validate the in silico target predictions. Plasmids containing wild-type and mutant Luc-STIM1 coding sequences were specifically synthesized and used for the luciferase reporter assays. These plasmids code for firefly luciferase and Renilla luciferase, which function as 
tracking genes. Luciferase activity assays were performed according to the manufacturer's protocols. Briefly, SW620 cells were seeded in six-well plates, co-transfected with the lentiviral constructs containing the miR-185 or vector control and the wild-type or mutated target gene 3'-UTR with Lipofectamine 2000 (Invitrogen). Firefly and Renilla luciferase activity levels were measured $48 \mathrm{~h}$ after the transfection using a Luc-Pair miR Luciferase Assay Kit (GeneCopoeia Inc., Rockville, MD, USA) according to the manufacturer's recommendations. Activity levels were normalized to Renilla luciferase. The results represent three independent experiments, each performed in triplicate.

A detailed description of the Materials and methods used in this study can be found in Supplementary Materials and methods.

\section{CONFLICT OF INTEREST}

The authors declare no conflict of interest.

\section{ACKNOWLEDGEMENTS}

This work was supported by National Basic Research Program of China (Grant nos: 2010CB732405 and 2010CB529300), National Natural Science Foundation of China (Grant nos: 81030044, 81272343, 81070326, 81172096, 81472300, 81472301, 81201632 and 30900675 ) and National Science and Technology Projects of China (Grant no.: 2014ZX09508002).

\section{REFERENCES}

1 Center MM, Jemal A, Smith RA, Ward E. Worldwide variations in colorectal cancer. CA Cancer J Clin 2009; 59: 366-378.

2 Markowitz SD, Bertagnolli MM. Molecular origins of cancer: molecular basis of colorectal cancer. N Engl J Med 2009; 361: 2449-2460.

3 Hanahan D, Weinberg RA. Hallmarks of cancer: the next generation. Cell 2011; 144: 646-674.

4 Christofori G. New signals from the invasive front. Nature 2006; 441: 444-450.

5 Jemal A, Siegel R, Ward E, Murray T, Xu J, Smigal C et al. Cancer statistics, 2006. CA Cancer J Clin 2006; 56: 106-130.

6 Zhao L, Wang H, Liu C, Liu Y, Wang X, Wang S et al. Promotion of colorectal cancer growth and metastasis by the LIM and SH3 domain protein 1. Gut 2010; 59: 1226-1235.

7 Berridge MJ, Bootman MD, Roderick HL. Calcium signalling: dynamics, homeostasis and remodelling. Nat Rev Mol Cell Biol 2003; 4: 517-529.

8 Parekh AB. Store-operated CRAC channels: function in health and disease. Nat Rev Drug Discov 2010; 9: 399-410.

9 Putney JW Jr. Capacitative calcium entry: sensing the calcium stores. J Cell Biol 2005; 169: 381-382.

10 Zhang SL, Yu Y, Roos J, Kozak JA, Deerinck TJ, Ellisman MH et al. STIM1 is a $\mathrm{Ca}^{2+}$ sensor that activates CRAC channels and migrates from the $\mathrm{Ca}^{2+}$ store to the plasma membrane. Nature 2005; 437: 902-905.

11 Feske S, Gwack Y, Prakriya M, Srikanth S, Puppel SH, Tanasa B et al. A mutation in Orai1 causes immune deficiency by abrogating CRAC channel function. Nature 2006; 441: 179-185.

12 Zhang SL, Yeromin AV, Zhang XH, Yu Y, Safrina O, Penna A et al. Genome-wide RNAi screen of $\mathrm{Ca}(2+)$ influx identifies genes that regulate $\mathrm{Ca}(2+)$ releaseactivated $\mathrm{Ca}(2+)$ channel activity. Proc Natl Acad Sci USA 2006; 103: 9357-9362.

13 Park CY, Hoover PJ, Mullins FM, Bachhawat P, Covington ED, Raunser S et al. STIM1 clusters and activates CRAC channels via direct binding of a cytosolic domain to Orai1. Cell 2009; 136: 876-890.

14 Lioudyno MI, Kozak JA, Penna A, Safrina O, Zhang SL, Sen D et al. Orai1 and STIM1 move to the immunological synapse and are up-regulated during $T$ cell activation. Proc Natl Acad Sci USA 2008; 105: 2011-2016.

15 Guo RW, Wang H, Gao P, Li MQ, Zeng CY, Yu Y et al. An essential role for stromal interaction molecule 1 in neointima formation following arterial injury. Cardiovasc Res 2009; 81: 660-668.

16 Varga-Szabo D, Braun A, Kleinschnitz C, Bender M, Pleines I, Pham M et al. The calcium sensor STIM1 is an essential mediator of arterial thrombosis and ischemic brain infarction. J Exp Med 2008; 205: 1583-1591.

17 Okabe $\mathrm{H}$, Hale TM, Kumon $\mathrm{H}$, Heaton JP, Adams MA. The penis is not protected---in hypertension there are vascular changes in the penis which are similar to those in other vascular beds. Int J Impot Res 1999; 11: 133-140.

18 Lu W, Wang J, Peng G, Shimoda LA, Sylvester JT. Knockdown of stromal interaction molecule 1 attenuates store-operated $\mathrm{Ca}^{2+}$ entry and $\mathrm{Ca}^{2+}$ responses to acute hypoxia in pulmonary arterial smooth muscle. Am J Physiol Lung Cell Mol Physiol 2009; 297: L17-L25.
19 Suyama E, Wadhwa R, Kaur K, Miyagishi M, Kaul SC, Kawasaki H et al. Identification of metastasis-related genes in a mouse model using a library of randomized ribozymes. J Biol Chem 2004; 279: 38083-38086.

20 Roberts-Thomson SJ, Peters AA, Grice DM, Monteith GR. ORAI-mediated calcium entry: mechanism and roles, diseases and pharmacology. Pharmacol Ther 2010; 127: $121-130$.

21 Yang S, Zhang JJ, Huang XY. Orai1 and STIM1 are critical for breast tumor cell migration and metastasis. Cancer Cell 2009; 15: 124-134.

22 Chen YF, Chiu WT, Chen YT, Lin PY, Huang HJ, Chou CY et al. Calcium store sensor stromal-interaction molecule 1-dependent signaling plays an important role in cervical cancer growth, migration, and angiogenesis. Proc Natl Acad Sci USA 2011; 108: $15225-15230$

23 Thiery JP, Acloque H, Huang RY, Nieto MA. Epithelial-mesenchymal transitions in development and disease. Cell 2009; 139: 871-890.

24 Thiery JP, Sleeman JP. Complex networks orchestrate epithelial-mesenchymal transitions. Nat Rev Mol Cell Biol 2006; 7: 131-142.

25 Gregory PA, Bert AG, Paterson EL, Barry SC, Tsykin A, Farshid G et al. The miR-200 family and miR-205 regulate epithelial to mesenchymal transition by targeting ZEB1 and SIP1. Nat Cell Biol 2008; 10: 593-601.

26 Kraljevic Pavelic S, Sedic M, Bosnjak H, Spaventi S, Pavelic K. Metastasis: new perspectives on an old problem. Mol Cancer 2011; 10: 22.

27 Loboda A, Nebozhyn MV, Watters JW, Buser CA, Shaw PM, Huang PS et al. EMT is the dominant program in human colon cancer. BMC Med Genom 2011; 4: 9.

28 Gulhati P, Bowen KA, Liu J, Stevens PD, Rychahou PG, Chen M et al. mTORC1 and mTORC2 regulate EMT, motility, and metastasis of colorectal cancer via RhoA and Rac1 signaling pathways. Cancer Res 2011; 71: 3246-3256.

29 Peinado H, Olmeda D, Cano A. Snail, Zeb and bHLH factors in tumour progression: an alliance against the epithelial phenotype? Nat Rev Cancer 2007; 7: 415-428.

$30 \mathrm{Hu}$ J, Qin K, Zhang Y, Gong J, Li N, Lv D et al. Downregulation of transcription factor Oct4 induces an epithelial-to-mesenchymal transition via enhancement of Ca2+ influx in breast cancer cells. Biochem Biophys Res Commun 2011; 411: 786-791.

31 Bartel DP. MicroRNAs: genomics, biogenesis, mechanism, and function. Cell 2004; 116: 281-297.

32 Zhang $\mathrm{H}$, Li Y, Lai M. The microRNA network and tumor metastasis. Oncogene 2010; 29: 937-948.

33 Gregory PA, Bracken CP, Bert AG, Goodall GJ. MicroRNAs as regulators of epithelial-mesenchymal transition. Cell cycle 2008; 7: 3112-3118.

34 Wang Z, Li Y, Ahmad A, Azmi AS, Kong D, Banerjee S et al. Targeting miRNAs involved in cancer stem cell and EMT regulation: an emerging concept in overcoming drug resistance. Drug Resist Updates 2010; 13: 109-118.

35 Zhuang R, Rao JN, Zou T, Liu L, Xiao L, Cao S et al. miR-195 competes with HuR to modulate stim 1 mRNA stability and regulate cell migration. Nucleic Acids Res 2013; 41: 7905-7919.

36 Liu X, Zhang Z, Sun L, Chai N, Tang S, Jin J et al. MicroRNA-499-5p promotes cellular invasion and tumor metastasis in colorectal cancer by targeting FOXO4 and PDCD4. Carcinogenesis 2011; 32: 1798-1805.

37 Duranton B, Holl V, Schneider Y, Carnesecchi S, Gosse F, Raul F et al. Polyamine metabolism in primary human colon adenocarcinoma cells (SW480) and their lymph node metastatic derivatives (SW620). Amino acids 2003; 24: $63-72$.

38 Singh A, Settleman J. EMT, cancer stem cells and drug resistance: an emerging axis of evil in the war on cancer. Oncogene 2010; 29: 4741-4751.

39 Lamouille S, Xu J, Derynck R. Molecular mechanisms of epithelial-mesenchymal transition. Nat Rev Mol Cell Biol 2014; 15: 178-196.

40 Prevarskaya N, Skryma R, Shuba Y. Calcium in tumour metastasis: new roles for known actors. Nat Rev Cancer 2011; 11: 609-618.

41 Yang S, Huang XY. $\mathrm{Ca}^{2+}$ influx through L-type $\mathrm{Ca}^{2+}$ channels controls the trailing tail contraction in growth factor-induced fibroblast cell migration. $J$ Biol Chem 2005; 280: 27130-27137.

42 Ritchie MF, Zhou Y, Soboloff J. WT1/EGR1-mediated control of STIM1 expression and function in cancer cells. Front Biosci 2011; 16: 2402-2415.

43 Stanisz H, Saul S, Muller CS, Kappl R, Niemeyer BA, Vogt T et al. Inverse regulation of melanoma growth and migration by Orai1/STIM2-dependent calcium entry. Pigment Cell Melanoma Res 2014; 27: 442-453.

44 Tan Z, Jiang H, Wu Y, Xie L, Dai W, Tang H et al. miR-185 is an independent prognosis factor and suppresses tumor metastasis in gastric cancer. Mol Cell Biochem 2014; 386: 223-231.

45 Zhi Q, Zhu J, Guo X, He S, Xue X, Zhou J et al. Metastasis-related miR-185 is a potential prognostic biomarker for hepatocellular carcinoma in early stage. Biomed Pharmacother 2013; 67: 393-398. 
46 Takahashi Y, Forrest AR, Maeno E, Hashimoto T, Daub CO, Yasuda J. MiR-107 and MiR-185 can induce cell cycle arrest in human non small cell lung cancer cell lines. PLoS One 2009; 4: e6677.

47 Liu M, Lang N, Chen X, Tang Q, Liu S, Huang J et al. miR-185 targets RhoA and Cdc42 expression and inhibits the proliferation potential of human colorectal cells. Cancer Lett 2011; 301: 151-160.

48 Tang H, Wang Z, Liu X, Liu Q, Xu G, Li G et al. LRRC4 inhibits glioma cell growth and invasion through a miR-185-dependent pathway. Curr Cancer Drug Targets 2012; 12: 1032-1042

49 Yoon JH, Choi YJ, Choi WS, Ashktorab H, Smoot DT, Nam SW et al. GKN1-miR-185-DNMT1 axis suppresses gastric carcinogenesis through regulation of epigenetic alteration and cell cycle. Clin Cancer Res 2013; 19: 4599-4610.

50 Imam JS, Buddavarapu K, Lee-Chang JS, Ganapathy S, Camosy C, Chen Y et al. MicroRNA-185 suppresses tumor growth and progression by targeting the Six 1 oncogene in human cancers. Oncogene 2010; 29: 4971-4979.
51 Akcakaya P, Ekelund S, Kolosenko I, Caramuta S, Ozata DM, Xie H et al. miR-185 and miR-133b deregulation is associated with overall survival and metastasis in colorectal cancer. Int J Oncol 2011; 39: 311-318.

(2) $(-)$ This work is licensed under a Creative Commons AttributionBY NC ND NonCommercial-NoDerivs 4.0 International License. The images or other third party material in this article are included in the article's Creative Commons license, unless indicated otherwise in the credit line; if the material is not included under the Creative Commons license, users will need to obtain permission from the license holder to reproduce the material. To view a copy of this license, visit http:// creativecommons.org/licenses/by-nc-nd/4.0/

(c) The Author(s) 2014

Supplementary Information accompanies this paper on the Oncogene website (http://www.nature.com/onc) 